05,06

\title{
Прямой и обратный магнитоэлектрический эффект в орторомбических монокристаллах $\mathrm{Dy}_{1-x} \mathrm{Ho}_{x} \mathrm{MnO}_{3}$
}

\author{
() А.Л. Фрейдман ${ }^{1,2}$, И.Н. Хороший ${ }^{2}$, М.И. Колков ${ }^{1}$, К.Ю. Терентьев ${ }^{1}$ \\ ${ }^{1}$ Институт фозики им. Л.В. Киренского СО РАН ФИЦ КНЦ СО РАН, \\ Красноярск, Россия \\ ${ }^{2}$ Сибирский фредеральный университет, \\ Красноярск, Россия \\ E-mail: fss4@yandex.ru
}

Поступила в Редакцию 3 августа 2021 г.

В окончательной редакции 3 августа 2021 г.

Принята к публикации 4 августа 2021 г.

Исследован магнитоэлектрический отклик в серии кристаллов $\mathrm{Dy}_{1-x} \mathrm{Ho}_{x} \mathrm{MnO}_{3}$, с замещением $x$ от 0 до 0.4. Результаты измерений показали, что по мере увеличения х наблюдается уменьшение как поляризации $P_{a}$, так и величины восприимчивости обратного магнитоэлектрического эффекта $\alpha_{b a}$. Кроме того, увеличивается поле $H_{b}$, при котором наблюдается переход $P_{c} \rightarrow P_{a}$, уменьшается диапазон температур существования магнитоэлектрического отклика. Таким образом, увеличение замещения $x$ подавляет магнитоэлектрический эффект в данном ряду соединений. Результаты измерений также косвенно указывают на пролонгацию состояния $P_{a}$, а, следовательно, и магнитного состояния с циклоидой, лежащей в плоскости $a b$, внешним электрическим полем $E_{a}$.

Ключевые слова: мультиферроики, магнитоэлектрический эффект, фазовый переход, сегнетоэлектричество.

DOI: 10.21883/FTT.2021.12.51673.185

\section{1. Введение}

В последние два десятилетия наблюдается быстрый рост количества публикаций, связанных с темой мультиферроиков. Мультиферроиками называются соединения, в которых присутствует хотя бы два из следующих трех типов спонтанного упорядочения: магнитное, сегнетоэлектричекое и сегнетоупругое [1]. Мультиферроики, в которых одновременно реализуется магнитное и сегнетоэлектрическое упорядочение, привлекают внимание ввиду наличия в них магнитоэлектрического эффекта, который заключается в связи магнитной подсистемы с электрической. Это означает что воздействуя на кристалл магнитным полем возможно изменение его электрической поляризации $P=P(H)$ и наоборот, при внесении кристалла во внешнее электрическое поле изменяется его магнитный момент $M=M(E)$. В последнее время в литературе устоялась терминология, согласно которой первый эффект называют прямым магнитоэлектрическим эффектом ( $\mathrm{ME}_{H}$-эффект), а второй обратным ( $\left.\mathrm{ME}_{E}-э ф ф е к т\right)$. Одновременное присутствие сегнетоэлектрического и магнитного упорядочения в кристалле приводит к возникновению новых интересных явлений, которые также перспективны и сточки зрения приложений.

Магнитоэлектрический эффект проявляет себя совершенно по-разному в различных соединениях. Так, например, эффект наблюдается и в соединениях, вовсе не являющихся мультиферроиками. Замечательный пример тому монокристаллы семейства парамагнитных боратов, где обнаружен гигантский магнитоэлектрический эф- фект [2,3]. Монокристаллы $\mathrm{HoAl}_{3}\left(\mathrm{BO}_{3}\right)_{4}$ обладают группой симметрии R32, которая запрещает пироэлектричество, но разрешает пьезоэлектрический эффект. Ввиду сильной одноионной анизотропии магнитное поле приводит к деформации решетки за счет магнитострикции, что посредством пьезоэлектрического эффекта влияет на поляризацию образца. При этом температуры, при которых наблюдается эффект соответствуют парамагнитному состоянию.

Тем не менее, согласно общим представлениям наибольших эффектов стоит ожидать от соединений, для которых величины диэлектрической и магнитной проницаемостей наибольшие, т. е. от мультиферроиков с ферромагнитным и сегнетоэлектрическим упорядочением $[4,5]$.

Мультиферроики принято разделять на два типа. Coединения, в которых сегнетоэлектрический переход и переход в упорядоченное магнитное состояние происходит независимо друг от друга, относят к мультиферроикам первого типа, если же сегнеоэлектрический переход является несобственным и вызван изменением магнитной структуры, то эти соединения относят к мультиферроикам второго типа. В несобственных сегнетоэлектриках первичный параметр порядка представляет собой моду искажения решетки, имеющую ненулевой волновой вектор, а спонтанная поляризация появляется как параметр вторичного порядка, индуцированный искажением решетки. Причиной к искажению решетки может являться, например, спонтанная магнитострикция, возникающая при магнитном фазовом переходе. Тип 
мультиферроика косвенно отражает величину магнитоэлектрического отклика. Действительно, в соединениях, где сама магнитная структура продуцирует возникновение нескомпенсированного электрического дипольного момента, следует ожидать сильное влияние магнитной подсистемы на поляризацию, и наоборот. Именно к этому классу мультифероиков относятся орторомбические перовскитоподобные манганиты некоторых редкоземельных элементов.

Орторомбические манганиты $\mathrm{RMnO}_{3}$ (пространственная группа $P b n m)$ привлекают огромный интерес исследователей ввиду богатой картины фазовых переходов в них. При температуре упорядочения ионов $\mathrm{Mn}^{3+}$ не происходит упорядочения редкоземельной подсистемы, она находится в парамагнитном состоянии, однако тип редкоземельного иона при этом оказывает определяющее влияние на тип упорядочения ионов $\mathrm{Mn}^{3+}$. В ряду редкоземельных элементов по мере уменьшения ионного радиуса $R^{3+}$ реализуется три участка соответствующие трем разным типам упорядочения $\mathrm{Mn}^{3+}$. Так для $R=\mathrm{La}-\mathrm{Eu}(Z=57-63)$ реализуется антиферромагнитное упорядочение А-типа, магнитные моменты ионов $\mathrm{Mn}^{3+}$ упорядочены ферромагнитно в $a b$-плоскости и антиферромагнитно вдоль оси $c$. В $\mathrm{HoMnO}_{3}(Z=67)$ возникает антиферромагнитный порядок Е-типа, когда магнитная структура в $a b$-плоскости строится по типу „вверх-вверх-вниз-вниз“ [6], что разрушает симметрию инверсии в этой плоскости.

В промежутке $Z=65-66$ в манганитах с редкоземельным элементом Tb и Dy по мере уменьшения температуры наблюдается целая серия магнитных переходов. При температуре Нееля $T_{N}\left(41 \mathrm{~K}\right.$ для $\mathrm{TbMnO}_{3}$ и $39 \mathrm{~K}$ для $\mathrm{DyMnO}_{3}$ ) возникает несоразмерное синусоидально-модулированное коллинеарное антиферромагнитное упорядочение магнитных моментов ионов $\mathrm{Mn}^{3+}$ с волновым вектором $(0, k, 1)$, где $k \sim 0.29$ для $\mathrm{TbMnO}_{3}$ и $k \sim 0.39$ для $\mathrm{DyMnO}_{3}[7,8]$.

Как было показано в работе [6] по мере уменьшения ионного радиуса редкоземельного элемента происходит эволюция искажения решетки $\mathrm{GdFeO}_{3}$-типа, которое заключается в подкручивании октаэдров $\mathrm{MnO}_{6}$. В результате этого изменяется длина путей, и, следовательно, энергия сверхобменных взаимодействий в $a b$-плоскости между ближайшими соседями по пути $\mathrm{Mn}-\mathrm{O}-\mathrm{Mn}$ (ферромагнитное упорядочение), а также взаимодействие еще по двум путям сверхобмена через два иона кислорода $\mathrm{Mn}-\mathrm{O}-\mathrm{O}-\mathrm{Mn}$, один из которых антиферромагнитный (вдоль $b$-оси), а другой слабый ферромагнитный (вдоль $а$-оси). При достижении определенной степени искажения решетки $\mathrm{GdFeO}_{3}$-типа (которое определяется ионным радиусом редкоземельного элемента) возникает конкуренция между ферромагнитным вкладом от ближайших соседей и антиферромагнитным от следующих по близости соседей. Последнее приводит к спиновой фрустрации и возникновению несоразмерного синусоидального магнитного упорядочения и антиферромагнетизма Е-типа.
В соединениях $\mathrm{TbMnO}_{3}$ и $\mathrm{DyMnO}_{3}$ в синусоидальной магнитной фазе не наблюдается возникновение спонтанной поляризации. Однако, по мере дальнейшего уменьшения температуры волновое число $k$ уменьшается вплоть до температуры $T_{S}$, ниже которой остается практически неизменным. В этой точке происходит переход, который сопровождается появлением спонтанной электрической поляризации направленной вдоль оси $c$. Сегнетоэлектрическая фаза возникает при температуре $T_{S}=28 \mathrm{~K}$ для $\mathrm{TbMnO}_{3}$ и $19 \mathrm{~K}$ для $\mathrm{DyMnO}_{3}$. В работе [9] проведено исследование по дифракции нейтронов на кристалле $\mathrm{TbMnO}_{3}$, которое показало, что магнитное упорядочение, соответствующее сегнетоэлектрической фазе является эллиптически модулированной циклоидной спиральной магнитной структурой, этот вывод также подтверждается работой [10]. Третий магнитный переход наблюдается при температурах $\sim 7$ и $\sim 5 \mathrm{~K}$ в $\mathrm{TbMnO}_{3}$ и $\mathrm{DyMnO}_{3}$, соответственно, при котором упорядочивается редкоземельная подсистема. Измерения магнитных характеристик также проведены в работе [11].

Ниже температуры $T_{S}$ спонтанная поляризация в этих соединениях описывается выражением $\mathbf{P} \propto\left[\left(\mathbf{r}_{i+1}-\mathbf{r}_{i}\right) \times\left[\mathbf{S}_{i} \times \mathbf{S}_{i+1}\right]\right.$, где $\mathbf{r}_{i}$ и $\mathbf{S}_{i}-$ радиус-вектор, проведенный до $i$-го иона $\mathrm{Mn}^{3+}$ и его спин, соответственно [12]. Как видно из этого выражения поляризация отлична от нуля только в том случае, если магнитные моменты соседних ионов $\mathrm{Mn}^{3+}$ неколлинеарны, чему соответствует спиновая циклоида. При этом направление результирующей поляризации оказывается перпендикулярно направлению модуляции магнитного момента $b$ и плоскости циклоиды. В нулевом магнитном поле в обоих соединениях возникает спонтанная поляризация, направленная вдоль оси $c$, то есть спиновая циклоида лежит в плоскости $b c$. Однако, при приложении внешнего магнитного поля в $a b$-плоскости наблюдается резкое изменение направления спонтанной поляризации $P_{c} \rightarrow P_{a}$, которое связано с опрокидыванием циклоиды моментов $\mathrm{Mn}^{3+}$ в плоскость $a b$. Последнее подтверждено экспериментально в работе [13]. Эффект переключения направления вектора спонтанной поляризации под действием внешнего магнитного поля очень привлекателен, так как восприимчивость поляризации к внешнему магнитному полю в точке перехода $P_{c} \rightarrow P_{a}$ крайне высока, а значит может представлять интерес для приложений.

В предыдущей нашей работе [14] было проведено исследование прямого и обратного магнитоэлектрического эффекта в $\mathrm{DyMnO}_{3}$. В данной работе мы сфокусировались на эволюции магнитоэлектрического отклика по мере замещения ионов $\mathrm{Dy}^{3+}$ ионами $\mathrm{Ho}^{3+}$ в монокристаллах состава $\mathrm{Dy}_{1-x} \mathrm{Ho}_{x} \mathrm{MnO}_{3}$ с $x=0-0.4$.

\section{2. Экспериментальная часть}

Монокристаллы $\mathrm{Dy}_{1-x} \mathrm{Ho}_{x} \mathrm{MnO}_{3}$ с величиной замещения $x=0-0.4$ были получены методом спонтанной кри- 
сталлизации из раствора в расплаве. Средний размер полученных монокристаллов составлял $\sim 1 \times 1 \times 1.5 \mathrm{~mm}^{3}$. Рентгеноструктурный анализ показал, что полученные монокристаллы $\mathrm{Dy}_{1-x} \mathrm{Ho}_{x} \mathrm{MnO}_{3}$ принадлежат к ромбической пространственной группе Pbnm. Всего в работе исследовано четыре образца с замещением $x=0$, $0.2,0.3$ и 0.4 .

Для измерений прямого и обратного магнитоэлектрических эффектов образцы подготавливались в форме плоского конденсатора путем шлифования. На подготовленные грани монокристалла, перпендикулярные кристаллографической оси $a$, наносились обкладки из токопроводящей пасты на эпоксидной основе с серебряным наполнителем.

Измерения электрической поляризации $\left(\mathrm{ME}_{H}\right.$-эффекта) были осуществлены путем измерения электрического заряда, стекающего с обкладок образца, с помощью электрометра Keithley 6517B. Перед измерениями образцы предварительно поляризовались путем охлаждения в постоянном электрическом поле $E_{0}=7.4 \mathrm{kV} / \mathrm{cm}$, приложенном вдоль оси $a$ кристалла до температуры $4.2 \mathrm{~K}$. После чего приложенное электрическое поле снималось, и начинался процесс измерений. Были проведены измерения температурных $P(T)$ и полевых $P(H)$ зависимостей поляризации $P_{a}$ вдоль оси $a$. Магнитное поле $H$ во время измерений поляризации прикладывалось вдоль $b$ направления.

Измерения обратного магнитоэлектрического эффекта $\left(\mathrm{ME}_{E}\right.$-эффект) были проведены на экспериментальной установке, собранной авторами. Подробное описание экспериментальной установки и метода измерений представлены в работах $[2,15,16]$. Также как и в случае $\mathrm{ME}_{H}$-эффекта, перед проведением измерений $\mathrm{ME}_{E}$-эффекта образец предварительно поляризовался путем охлаждения в постоянном электрическом поле $E=7.4 \mathrm{kV} / \mathrm{cm}$, приложенном вдоль оси $a$ кристалла. После охлаждения поляризующее поле убиралось, и к образцу прикладывалось переменное электрическое поле $\mathbf{E}=e_{0} \cos (\omega t)$ с амплитудой $e_{0}=3.7 \mathrm{kV} / \mathrm{cm}$ и частотой $f=1 \mathrm{kHz}$. Из-за магнитоэлектрического эффекта приложенное переменное электрическое поле $E$ изменяет магнитный момент образца по периодическому закону. В результате, в съемной катушке индуцируется напряжение, которое в дальнейшем, на основе калибровочных данных, интерпретируется как амплитуда колебаний магнитного момента образца.

Возбуждающее электрическое поле было приложено только вдоль $a$-оси, при этом изменение магнитного момента $\Delta M$ фиксировалось вдоль оси $b$ (поперечный $\mathrm{ME}_{E}$-эффект). Для измерения сигнала использовался синхронный усилитель SR830, фиксирующий как первую гармонику колебаний магнитного момента $\Delta M^{\prime}$, так и вторую $\Delta M^{\prime \prime}$. Первая из них соответствует колебанию магнитного момента на частоте возбуждающего электрического поля $\omega$, а вторая - колебанию магнитного момента на удвоенной частоте $2 \omega$. Как было показано в работе [16], $\Delta M^{\prime}=\alpha e_{0}, \Delta M^{\prime \prime}=\beta e_{0}^{2} / 2$, где $\alpha$ и $\beta-$ восприимчивости линейного и квадратичного членов обратного магнитоэлектрического эффекта по электрическому полю, $e_{0}$ - амплитуда колебаний приложенного электрического поля. В данной работе приведены результаты по измерению только первой гармоники, однако, отметим, что в соединениях $\mathrm{Dy}_{1-x} \mathrm{Ho}_{x} \mathrm{MnO}_{3}$ возбуждалась также и вторая гармоника обратного магнитоэлектрического эффекта, что является темой для исследования в ближайшем будущем.

\section{3. Результаты и обсуждение}

В орторомбическом манганите $\mathrm{DyMnO}_{3}$ возникает спонтанная поляризация при температуре $T_{S}$, направленная вдоль оси $c$. Ввиду того, что форма и размер выросших кристаллов препятствовали подготовке образцов в виде плоского конденсатора с плоскостью перпендикулярной оси $c$, мы провели измерения магнитоэлектрических свойств вдоль направления $a$. Несмотря на то, что спонтанная поляризация в данном соединении возникает вдоль $c$, она может быть опрокинута в направлении а внешним магнитным полем $H_{b}$. Явным маркером перехода $P_{c} \rightarrow P_{a}$ является резкое увеличение диэлектрической проницаемости $\varepsilon_{a}$ при появлении спонтанной поляризации $P_{a}$. На рис. $1, a$ представлены зависимости относительной диэлектрической проницаемости $\varepsilon_{a}$ от величины приложенного магнитного поля $H_{b}$. Явные аномалии наблюдаются для соединений $\mathrm{Dy}_{1-x} \mathrm{Ho}_{x} \mathrm{MnO}_{3}$ с величиной замещения $x=0.2$ и 0.3 , для $x=0.4$ аномалия уже практически не заметна, для $x=0$ данные можно посмотреть в работе [8]. По мере увеличения замещения $x$ в соединении, магнитное поле, приводящее к переориентации вектора поляризации увеличивается. При $x=0 H_{c} \sim 13 \mathrm{kOe}(T=2 \mathrm{~K})$ [8], для $x=0.2,0.3$ поле, при котором наблюдается аномалия, составляет $H_{c} \sim 19,22 \mathrm{kOe}(T=4.2 \mathrm{~K})$, соответственно. Для $x=0.4$ поле $H_{c}$ так же около $22 \mathrm{kOе}$. Также на графиках зависимости $\Delta \varepsilon_{a}\left(H_{b}\right)$ явно виден гистерезис, который связан с последовательностью переходов $P_{c} \rightarrow P_{a} \rightarrow P_{c}$. Стрелками на рисунке показано направление изменения поля. Аномалиям диэлектрической проницаемости соответствует переход, заключающийся в опрокидывании плоскости циклоиды спинов ионов $\mathrm{Mn}^{3+}$ от $b c$ к $b a$ с одновременным поворотом вектора спонтанной поляризации $P_{c} \rightarrow P_{a}$.

На рис. $1, b$ показаны зависимости электрической поляризации от внешнего магнитного поля $H_{b}$. Часть из приведенных данных уже была нами опубликована ранее [14] для чистого кристалла $\mathrm{DyMnO}_{3}$, в данной работе мы дополняем их и приводим более полную картину с замещением Dy на Но. Как видно из зависимостей $P(H)$ по мере увеличения замещения $x$ происходит уменьшение величины поляризации, кроме того также увеличивается и магнитное поле перехода $P_{c} \rightarrow P_{a}$. В целом можно утверждать, что графики поляризации полностью согласуются с зависимостями $\varepsilon(H)$. Что 


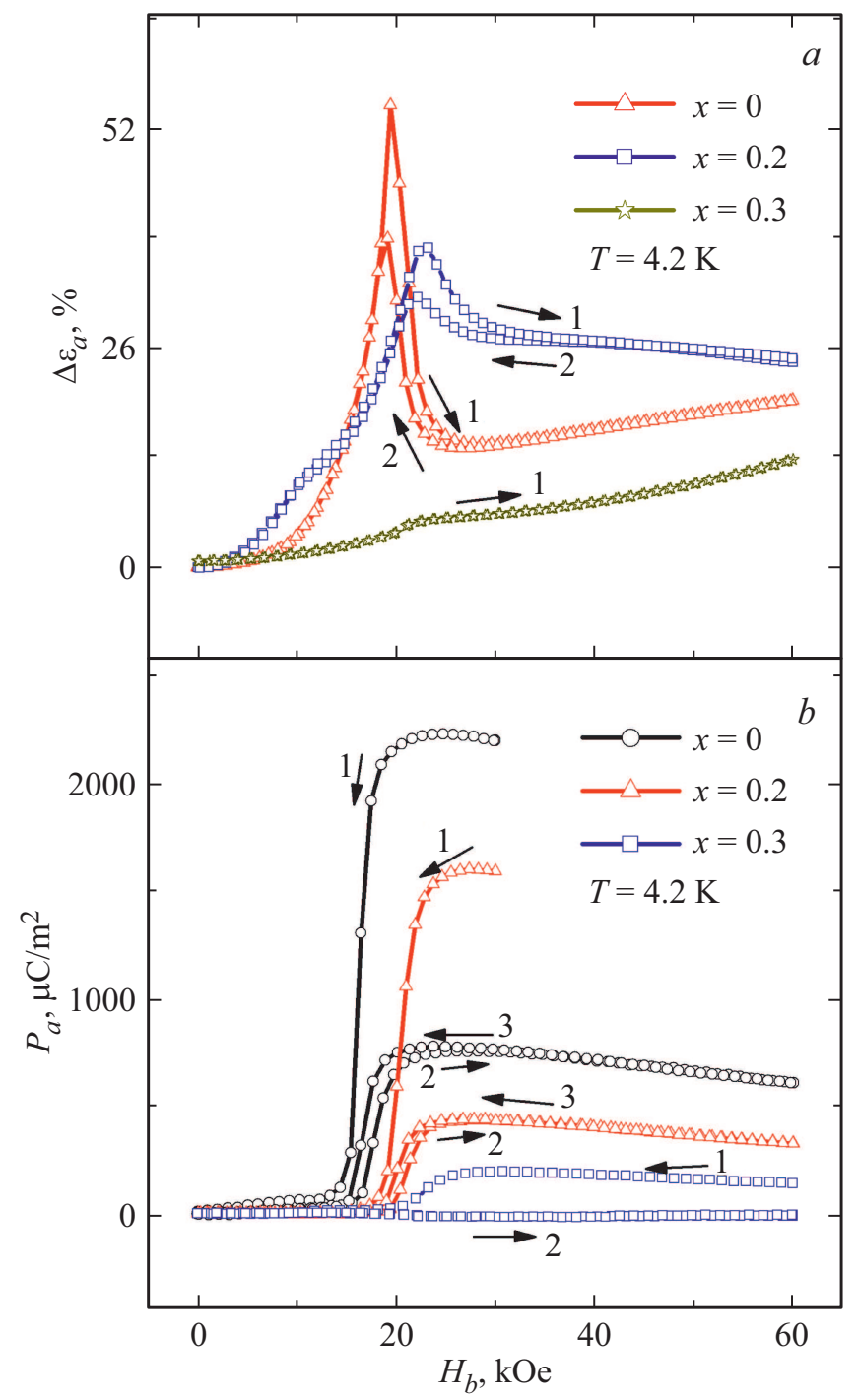

Рис. 1. Зависимость относительного изменения диэлектрической проницаемости $\Delta \varepsilon(a)$ и поляризации $P_{a}(b)$ от внешнего магнитного поля $H_{b}$ для разных составов $\mathrm{Dy}_{1-x} \mathrm{Ho}_{x} \mathrm{MnO}_{3}$ $(x=0-0.4)$ при температуре $T=4.2 \mathrm{~K}$.

касается поляризации для $x=0.4$, то такие измерения были нами проведены, однако величины измеренной поляризации были слишком малы, чтобы достоверно утверждать о наличии эффекта.

Обращает на себя внимание поляризация образца с $x=0.3$. Начальный ход кривой был сделан, как и для всех остальных кривых, с предохлаждения в магнитном и электрическом поле. Магнитное поле $H_{b}$ при предохлаждении было приложено для инициации перехода в состояние $P_{a}$, электрическое поле $E_{a}$ было приложено для того чтобы сегнетоэлектрическое состояние $P_{a}$ было монодоменным к началу измерений поляризации. Процесс измерений начинался с уменьшения магнитного поля в точке старта соответствующей магнитному полю, приложенному при предохлаждении, этот ход обозначен цифрой 1 на графиках рис. 1, $b$. Закономер- но, что при уменьшении магнитного поля происходит резкий спад поляризации в точке перехода $P_{a} \rightarrow P_{c}$, при последующем увеличении магнитного поля снова инициируется состояние $P_{a}$, однако величина поляризации не столь высока, так как в процессе данного перехода $P_{c} \rightarrow P_{a}$ внешнее электрическое поле, способствующее образованию монодоменного состояния, уже не было приложено. Однако в случае с $x=0.3$ наблюдается не только уменьшение величины поляризации при повторной инициации состояния $P_{a}$, но и изменение ее знака. Последнее означает, что в данном образце существует некоторое предпочтительное направление поляризации вдоль $a$, противоположное электрическому полю, приложенному в процессе предохлаждения. Вероятно, это особенность конкретно взятого образца и не относится ко всем соединения $\mathrm{Dy}_{1-x} \mathrm{Ho}_{x} \mathrm{MnO}_{3}$ с $x=0.3$. Например, одной из возможных причин этому может быть градиент внутренних напряжений в кристалле. Согласно теории фазовых переходов возникновение параметра порядка равновероятно как в направлении $+a$, так и $-a$, однако дополнительные полярные внешние факторы могут привести к тому, что энергия одного из направлений окажется меньше. Последнее, конечно, не гарантирует монодоменное состояние, так как необходимо также учитывать и поверхностную энергию сегнетоэлектрика, которая минимизируется путем разбиения объема кристалла на домены с различным направлением сегнетоэлектрической поляризации.

В случае обратного магнитоэлектрического эффекта, когда к кристаллу прикладывалось возбуждающее переменное электрическое поле и измерялись осцилляции магнитного момента, зависимость от предыстории охлаждения образца так же наблюдалась. При возбуждении $\mathrm{ME}_{E}$-эффекта к кристаллу прикладывается переменное электрическое поле, при этом возникает пьезоэлектрический эффект, знак которого определяется взаимным направлением вектора спонтанной поляризации и внешнего возбуждающего электрического поля. Если кристалл разбит на сегнетоэлектрические домены, то неизбежно возникновение как положительного, так и отрицательного пьезоэффекта в различных областях кристалла. Не вникая в тип деформации, последнее означает, что возникает как положительная, так и отрицательная деформация, которые по-разному влияют на макроскопический магнитный момент, и вероятно, противоположно. Поэтому результирующий $\mathrm{ME}_{E}$-эффект мал, когда измерения проводились без процедур приводящих к монодоменному состоянию $P_{a}$.

Наиболее интересную зависимость от предыстории демонстрирует образец с $x=0.3$. Как было сказано ранее, в данном образце повторное индуцирование перехода $P_{c} \rightarrow P_{a}$ приводит к возникновению поляризации $P_{a}$, знак которой противоположен той, что первоначально задана внешним полем. На рис. 2, а показана зависимость $\alpha_{b a}\left(H_{b}\right)$ для $x=0.3$, которая также демонстрирует изменение знака обратного магнитоэлектрического эффекта при повторном индуцировании состояния $P_{a}$. 
Восприимчивость $\alpha$ была определена как отношение амплитуды колебания намагниченности $\Delta M$ к амплитуде возбуждающего электрического поля $E, \alpha=\Delta M / E$. При этом фиксировались только те колебания намагниченности, которые осциллируют с той же частотой $(1 \mathrm{kHz})$, что и приложенное электрическое поле. Как видно из рисунка, в начальном ходе 1 магнитоэлектрический эффект имеет максимум в районе $20 \mathrm{kOe}$, который соответствует переходу $P_{a} \rightarrow P_{c}$, и пропадает в поле $H=0$. Однако, при увеличении магнитного поля в точке перехода $P_{c} \rightarrow P_{a}$ восприимчивость $\alpha_{b a}$ меняет свой знак и возникает минимум. При дальнейшем циклировании по магнитному полю отрицательный характер зависимости сохраняется.

На рис. $2, b$ представлены зависимости восприимчивости линейного по электрическому полю члена магнитоэлектрического эффекта $\alpha$ от магнитного поля $H_{b}$,

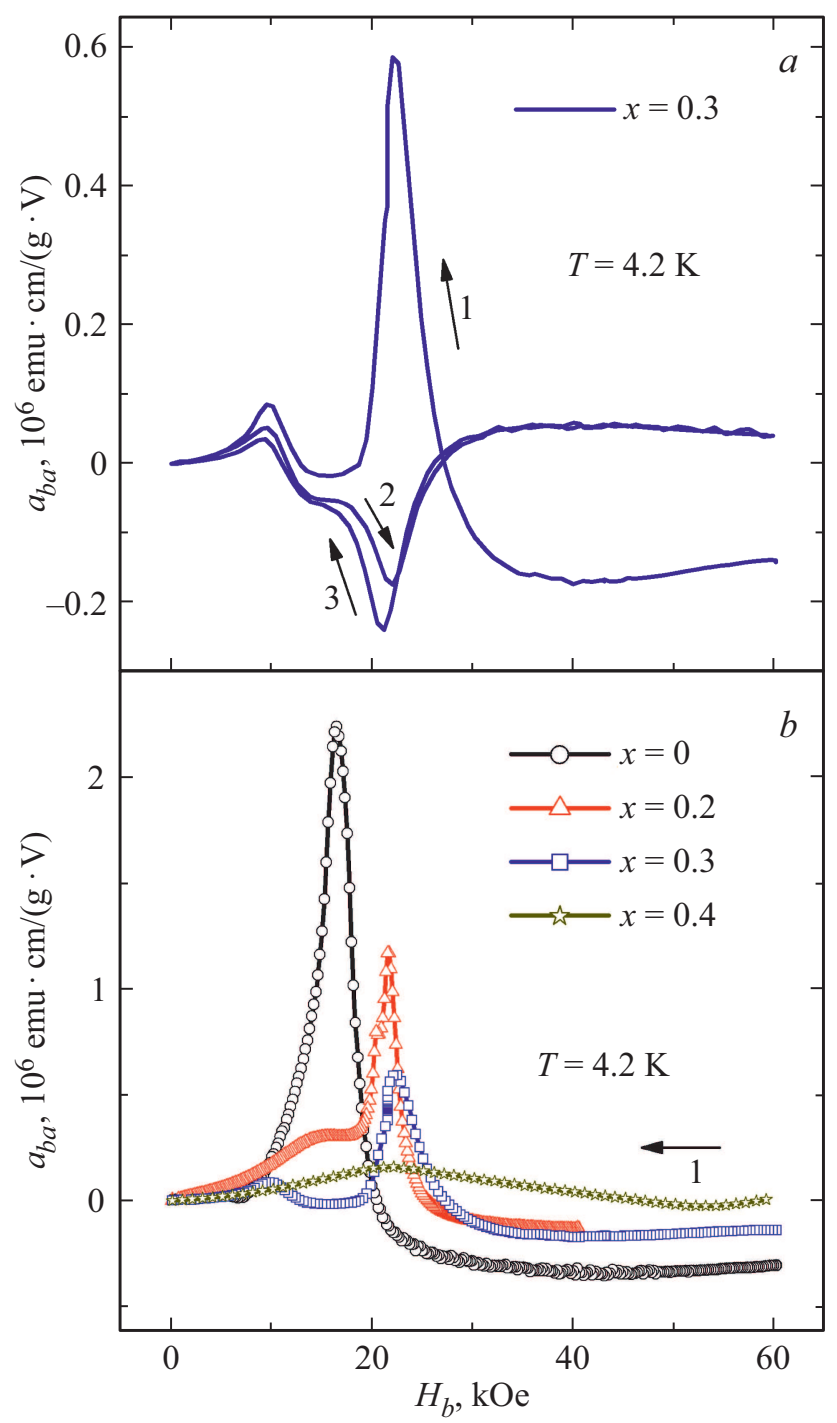

Рис. 2. Восприимчивость линейного члена магнитоэлектрического эффекта $\alpha_{b a}$ в зависимости от внешнего магнитного поля $H_{b}$ в $\mathrm{Dy}_{1-x} \mathrm{Ho}_{x} \mathrm{MnO}_{3} \mathrm{c} x=0.3(a)$ и другими составами $(b)$ при $T=4.2 \mathrm{~K}$. измеренные для различных величин замещения $x$ при $T=4.2 \mathrm{~K}$. Как и в случае поляризации, наиболее сильный эффект проявляется для незамещенного образца $\mathrm{DyMnO}_{3}$. Результаты измерений $\mathrm{ME}_{E}$-эффекта подтверждают, что по мере увеличения замещения $x$ магнитоэлектрический отклик ослабевает, а также увеличивается поле перехода $P_{c} \rightarrow P_{a}$, что согласуется с результатами измерений $P(H)$. Нам также удалось зафиксировать достоверный сигнал, относящийся к $\mathrm{ME}_{E}$-эффекту для образца с $x=0.4$, чего не удалось сделать при измерении $\mathrm{ME}_{H}$-эффекта, это говорит о большей чувствительности метода.

Температурные зависимости поляризации $P_{a}(T)$ и обратного магнитоэлектрического эффекта $\alpha_{b a}(T)$ показаны на рис. $3, a$ и $b$, соответственно. Эти измерения были проведены для различных величин замещения $x$ в магнитном поле $H_{b}=30 \mathrm{kOe}$. По мере увеличения

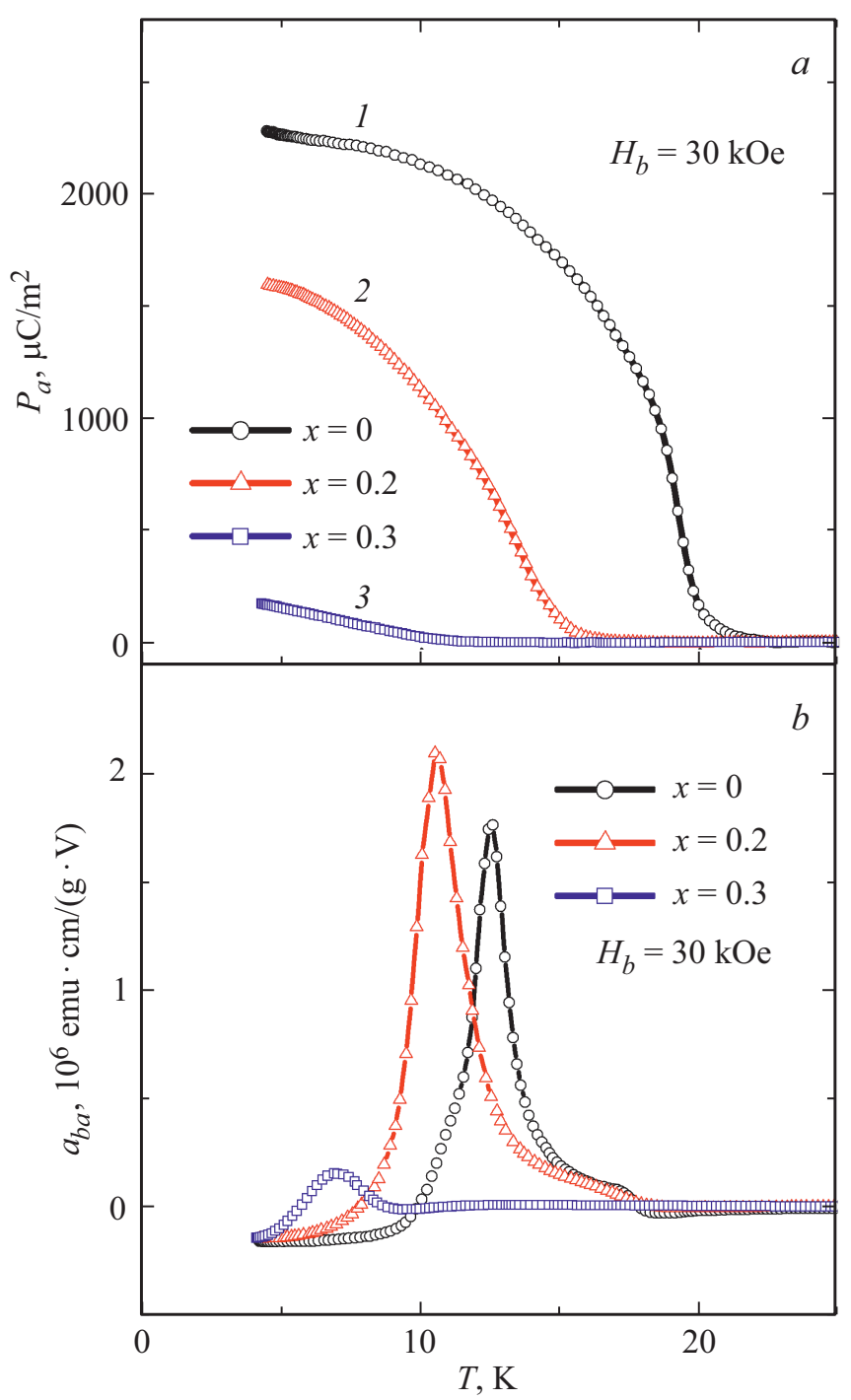

Рис. 3. Зависимость электрической поляризации $P_{a}(a)$ и восприимчивости линейного члена магнитоэлектрического эффекта $\alpha_{b a}(b)$ от температуры для различных замещений $x$ в поле $30 \mathrm{kOe}$. 
замещения уменьшается температура возникновения поляризации, что говорит о смещении температуры перехода $T_{s}$, соответствующего магнитному упорядочению типа циклоиды. Как известно при $x=1 \quad\left(\mathrm{HoMnO}_{3}\right)$ возникает упорядочение магнитных моментов марганцевой подсистемы Е-типа [17], представленные данные закономерно отражают процесс подавления соразмерной циклоидной фазы.

Подобная зависимость также проявляется и в $\mathrm{ME}_{E}$-эффекте, как видно из рис. $3, b$, однако эффект для образца с замещением $x=0.2$ полностью пропадает в тех же самых температурах, что и в незамещенном образце. Последнее противоречит данным $P_{a}(T)$, и, по всей видимости, обусловлено наличием возбуждающего электрического поля, которое присутствует в процессе измерения $\mathrm{ME}_{E}$-эффекта $(e=3.7 \mathrm{kV} / \mathrm{cm}, f=1 \mathrm{kHz})$. Действительно, хорошо известно, что в присутствии магнитного поля магнитный фазовый переход размывается, аналогичное размытие может происходить и для сегнетоэлектрика во внешнем электрическом поле. При наличии спонтанной поляризации энергия сегнетоэлектрика включает в себя член пропорциональный произведению спонтанной поляризации и напряженности электрического поля, -(PE), а, следовательно, системе становится энергетически выгодно поддерживать поляризацию в том случае, если электрическое поле не равно нулю. Это же означает, что при уменьшении амплитуды возбуждающего электрического поля максимальная температура, при которой наблюдается $\mathrm{ME}_{E}$-эффект, может уменьшиться. Более того, ввиду связи магнитного и сегнетоэлектрического порядков, можно ожидать, что электрическое поле $E_{a}$ удерживает магнитное упорядочение соответствующее спиновой циклоиде, лежащей в плоскости $a b$.

Как было сказано выше, при определении восприимчивости $\alpha_{b a}$ предполагалась линейная зависимость амплитуды колебания намагниченности $\Delta M$ от приложенного электрического поля $e$ при фиксации ее на частоте возбуждения. Такой подход основывается на предположении, что сама величина восприимчивости $\alpha_{b a}$ не зависит от амплитуды электрического поля, однако ввиду последних наблюдений, это может оказаться не так, в особенности вблизи фазового перехода. Поэтому мы планируем в ближайшем будущем более подробно исследовать петли сегнетоэлектрического гистерезиса и области возможных нелинейностей $\alpha_{b a}$ для тех же составов. Отдельный интерес также представляет вторая гармоника $\mathrm{ME}_{E}$-эффекта, то есть член квадратичный по электрическому полю.

\section{4. Заключение}

В работе проведено исследование магнитоэлектрического отклика в серии замещений $\mathrm{Dy}_{1-x} \mathrm{Ho}_{x} \mathrm{MnO}_{3}, \mathrm{c} x$ от 0 до 0.4. Исследованы температурные и полевые зависимости прямого и обратного магнитоэлектрическо- го эффекта. В целом можно заключить, что замещение ионов $\mathrm{Dy}^{3+}$ на $\mathrm{Ho}^{3+}$ оказывает подавляющее влияние на магнитоэлектрический эффект в данном соединении. По мере увеличения замещения $x$, наблюдается уменьшение поляризации $P_{a}$, уменьшение величины восприимчивости обратного магнитоэлектрического эффекта $\alpha_{b a}$, кроме того, увеличивается поле $H_{b}$, при котором наблюдается переход $P_{c} \rightarrow P_{a}$, уменьшается диапазон температур существования магнитоэлектрического отклика.

Результаты измерений также указывают на то, что приложенное электрическое поле способно пролонгировать состояние $P_{a}$, а, следовательно, и магнитное состояние с циклоидой, лежащей в плоскости $a b$. Также полученные данные указывают на возможное наличие нелинейных эффектов, связанных с зависимостью от электрического поля восприимчивости линейного члена магнитоэлектрического эффекта.

\section{Финансирование работы}

Исследование выполнено при финансовой поддержке РФФИ, Правительства Красноярского края и Красноярского краевого фонда науки в рамках научного проекта № 20-42-243008.

\section{Конфликт интересов}

Авторы заявляют, что у них нет конфликта интересов.

\section{Список литературы}

[1] W. Eerenstein, N.D. Mathur, J.F. Scott. Nature 442, 759 (2006).

[2] A.L. Freydman, A.D. Balaev, A.A. Dubrovskiy, E.V. Eremin, V.L. Temerov, I.A. Gudim. J. Appl. Phys. 115, 174103 (2014).

[3] K.-C. Liang, R.P. Chaudhury, B. Lorenz, Y.Y. Sun, L.N. Bezmaternykh, V.L. Temerov, C.W. Chu. Phys. Rev. B. 83, 180417(R) (2011).

[4] M. Fiebig. J. Phys. D 38, R123 (2005).

[5] И.Е. Чупис. Физика низких температур 36, 6, 597 (2010).

[6] T. Kimura, S. Ishihara, H. Shintani, T. Arima, K.T. Takahashi, K. Ishizaka, Y. Tokura. Phys. Rev. B 68, 060403(R) (2003).

[7] S. Quezel, F. Tcheou, J. Rossat-Mignod, G. Quezel, E. Roudaut. Physica 86-88B, 916 (1977).

[8] T. Kimura, G. Lawes, T. Goto, Y. Tokura, A. P. Ramirez. Phys. Rev. B 71, 224425 (2005).

[9] M. Kenzelmann, A. Harris, S.H. Jonas, C.L. Broholm, J. Schefer, S. Kim, C. Zhang, S. Cheong, O.P. Vajk, J.W. Lynn. M. Phys. Rev. Lett. 95, 087206 (2005).

[10] T. Arima, A. Tokunaga, T. Goto, H. Kimura, Y. Noda, Y. Tokura. Phys. Rev. Lett. 96, 097202 (2006).

[11] S.V. Semenov, M.I. Kolkov, K.Y. Terent'ev, N.S. Pavlovskiy, M.S. Pavlovskiy, A.D. Vasiliev, A.V. Shabanov, K.A. Shaykhutdinov, D.A. Balaev. J. Supercond. Nov. Magn. 32, 3315 (2019).

[12] H. Katsura, N. Nagaosa, A.V. Balatsky. Phys. Rev. Lett. 95, 057205 (2005). 
[13] N. Aliouane, K. Schmalzl, D. Senff, A. Maljuk, K. Prokes, M. Braden, D.N. Argyriou. Phys. Rev. Lett. 102, 207205 (2009).

[14] A.L. Freidman, S.V. Semenov, M.I. Kolkov, K.Yu. Terent'ev, N.S. Pavlovskiy, D.M. Gokhfeld, K.A. Shaykhutdinov, D.A. Balaev. J. Appl. Phys. 128, 094102 (2020).

[15] А.Д. Балаев, А.Л. Фрейдман. Поверхность. Рентген., синхротрон. и нейтрон. исслед. 1, 20 (2014).

[16] A.L. Freydman, D.A. Erofeev, V.L. Temerov, I.A. Gudim. J. Appl. Phys. 124, 134101 (2018).

[17] A. Munoz, M.T. Casais, J.A. Alonso, M.J. Martınez-Lope, J.L. Martıne, M.T. Fernandez-Diaz. Inorg. Chem. 40, 1020 (2001).

Редактор Т.Н. Василевская 